\title{
Research Progress on Regulatory T Cells and Their Relationship with Autoimmune Diseases
}

\author{
Cuicui Wang ${ }^{1}$, Zhaoxin $\mathrm{Mu}^{2}$, Zhenjiang $\mathrm{Hou}^{2, *}$ \\ ${ }^{1}$ Department of Medical Technology, Cangzhou Medical College, Cangzhou, China \\ ${ }^{2}$ Institute of Thyroid Diseases Affiliated to Cangzhou Medical College, Cangzhou Thyroid Disease Engineering Technology Research Center, \\ Cangzhou, China
}

Email address:

houzhenjiang@sina.com (Zhenjiang Hou)

*Corresponding author

To cite this article:

Cuicui Wang, Zhaoxin Mu, Zhenjiang Hou. Research Progress on Regulatory T Cells and Their Relationship with Autoimmune Diseases. Science Journal of Public Health. Vol. 8, No. 3, 2020, pp. 83-91. doi: 10.11648/j.sjph.20200803.14

Received: April 11, 2020; Accepted: May 29, 2020; Published: June 4, 2020

\begin{abstract}
Regulatory T cells (Tregs) are a subset of T cells with immunosuppressive functions. They secrete IL-10, TGF-âand other suppressive cytokines and direct contact among cells to suppress the autoimmunity response of effector $\mathrm{T}$ cells, promote immune tolerance, which has a very important role in maintaining the body's homeostasis. Foxp3 is a specific transcription factor of Treg cells and plays a key role in the regulation of Treg cell development and function. Treg and Th17 cells are derived from the differentiation of the same type of initial $\mathrm{CD} 4^{+} \mathrm{T}$ cells. The direction of differentiation depends on the type of cytokines in the environment. The functions of them are opposite to each other and transform each other under certain conditions. It plays an important role in inducing immune tolerance and preventing the occurrence of autoimmune diseases (AID). Once immune imbalance, it will lead to the occurrence of immune-related diseases. The decrease of its number or function will affect the immune state of the body, cause disorders of autoimmune tolerance and lead to the occurrence of AID. This article reviews the research of Treg and their cytokines in AID in order to understand the role of Treg in the pathogenesis of AID, and open a new way for the treatment of AID.
\end{abstract}

Keywords: Regulatory T Cells, Interleukin-10, Transforming Growth Factor-â, Forkhead Box p3, Autoimmune Diseases

\section{Introduction}

AID is a disorder of the body's immune function, with the decrease of immune tolerance to self-antigens and the production of a large amount of autoantibodies and immune complexes, resulting in a large class of diseases characterized by damage to multiple organs. Its disease spectrum is broad, mainly including more than 70 kinds of rheumatoid arthritis, systemic lupus erythematosus, etc. with different clinical manifestations. The exact etiology and pathogenesis are not yet clear. The prevalence rate has risen in recent years, which has seriously affected the labor ability and quality of life of patients and has become an important public health issue [1]. Treg cells are a type of $\mathrm{T}$ cells with immunosuppressive effects. Their function is mainly to inhibit the activation of other $\mathrm{T}$ cells, so they play a very important role in preventing the occurrence of AID. Since Sakaguchi's discovery, it has been a research hotspot in the field of immunology. In recent years, clinical research on Treg cells in rheumatoid arthritis, systemic lupus erythematosus and other AIDs has been widely carried out clinically, and certain progress has been achieved. This article summarizes the research of Treg cells and their role in AID in order to deepen the understanding of Treg cell differentiation regulation and the role of Treg cells in the pathogenesis of AID, which will open up a new way for the prevention and treatment of AID.

\section{Differentiation Regulation of Treg Cells}

Both Treg and Th17 cells are derived from the same type of initial $\mathrm{CD}^{+} \mathrm{T}$ cells. After differentiation into intermediate cells, the direction of their differentiation mainly depends on the type of cytokines in the environment, and they transform into each other under certain conditions. TGF- $\beta$ mediates the 
differentiation of T cells into Treg. After the addition of IL-6, the differentiation is stopped, and goes to Th17 direction. Treg cell differentiation is inhibited to a certain extent, which indicates that IL-6 plays a decisive role in the direction of T cell differentiation. Large amounts of IL-6 and IL-21 can also transform differentiated Treg cells into Th17 cells. Another study shows that TGF- $\beta$ plays an important role in the differentiation of $\mathrm{T}$ cells into Th17 or Treg cells [2]. Low levels of TGF- $\beta$ and IL- 6 together promote the differentiation of Th17 cells. High concentrations of TGF- $\beta$ acting alone can induce high Foxp3 expression, promote Treg cell differentiation, and cause $\mathrm{CD} 4{ }^{+} \mathrm{CD} 25^{+}$Treg cells to inhibit Th17. IL-17 and IL-6 can effectively inhibit the function of Th1 and transform Treg cells to Th17 cells. In the presence of IL-10, Th17 cells can be transformed into Treg cells. The differentiation of Treg cells is regulated by many factors. Under neutral conditions in vitro, TGF- $\beta$ will tend to balance towards the development of functional FoxP ${ }^{+}$Tregs. In the presence of inflammatory cytokines such as IL-1 $\beta$, IL-2, IL-15, functional Tregs are transformed into non-functional Tregs which produce IL-17. IL-2 is a key factor in regulating the homeostasis of Treg cells, and immature Treg cells can be used to increase the expression of Foxp3 and CD25 and promote differentiation into mature Treg cells. IL-2 and TGF- $\beta$ induce the differentiation of naive T cells into Treg cells. IL-2 induces STAT5, enhances Foxp3 expression, and promotes Treg differentiation. TGF- $\beta$ is mainly involved in the differentiation process of Treg through STAT5 induction, and retinoic acid activates the differentiation process by promoting TGF- $\beta$ and inducing Foxp3 [3]. IL-35 is a new anti-inflammatory factor secreted by Treg cells after IL-10 and TGF- $\beta$. It has stronger immunological functions than IL-10 and TGF- $\beta$ and plays a negative immunoregulatory role in the differentiation of Treg cells [3]. IL-35 can inhibit the proliferation of $\mathrm{T}$ cells, expand Treg cells, and induce iTr35 with stronger inhibitory activity to exert its immunomodulatory effect [4]. iTr35 can secrete IL-35 specifically, inhibit the proliferation of $\mathrm{T}$ cells, and is a key factor in inducing infectious tolerance, effectively preventing the occurrence and development of inflammation [5]. The differentiation state of $\mathrm{CD}^{+} \mathrm{T}$ cells is mainly determined by environmental factors and their transcription factors [6]. Under the stimulation of different cytokines, $\mathrm{CD} 4{ }^{+} \mathrm{T}$ cells can be differentiated into different effector T cells. The latter and Treg restrict each other in the process of function and differentiation, and jointly maintain the immune homeostasis of the body. Once the immune imbalance, it will lead to the occurrence of immune-related diseases $[6,7]$.

\section{Treg Cells and Autoimmune Diseases}

AID is a disorder of the body's immune function, reduced immune tolerance to self-antigens, and produces a large amount of autoantibodies and immune complexes, resulting in a large class of diseases characterized by damage to multiple organs. Its disease spectrum is broad, mainly including more than 70 kinds of rheumatoid arthritis, systemic lupus erythematosus, etc, with different clinical manifestations. The exact etiology and pathogenesis are not yet clear. The prevalence rate has risen in recent years, which has seriously affected the labor ability and quality of life of patients and has become an important public health issue [1]. Treg cells are a type of T cells with immunosuppressive effects. Their function is mainly to inhibit the activation of other T cells, so they play a very important role in preventing the occurrence of AID. Since Sakaguchi's discovery, it has been a research hotspot in the field of immunology. In recent years, clinical research on Treg cells in rheumatoid arthritis, systemic lupus erythematosus and other AIDs has been widely carried out clinically, and certain progress has been achieved.

\subsection{Treg Cells and Rheumatoid Arthritis (Rheumatoid Arthritis, $R A$ )}

RA is a common inflammatory autoimmune disease, mainly manifested as chronic progressive and invasive inflammation of the synovium of the joint, which leads to the destruction of bone and cartilage tissues through inflammatory cells, resulting in high morbidity, disability and mortality. Traditionally, Th1 / Th2 imbalance is the key factor leading to RA. Th1 cells in the synovial fluid of RA patients increased, and secreted cytokines such as IFN- $\gamma$, causing local chronic inflammation and matrix destruction of the joint synovium, and inhibited Th2 cell expression, leading to Th2 cell defects [8]. Research results on Treg cells in RA patients are controversial. Attridge and other researchers believed that a variety of cytokines can affected the suppressive function of Treg cells. For example, IL-21 secreted by Th17 cells made Teff resist the inhibitory effect of Treg cells. TNF- $\alpha$ was the main cytokine that affects the function of Treg cells in RA patients. Too much TNF- $\alpha$ can down-regulated the phosphorylation level of Foxp3, thereby inhibiting the function of Treg cells, so Treg cells cannot completely effectively inhibited the inflammatory response of RA patients [9]. Disturbance of Foxp3 methylation and CTLA-4 expression and loss of function in the enhancer region upstream of the locus can also cause defects in Treg cell function. In early RA patients, the level of synovium, synovial fluid and plasma IL-21 increased, which promoted the formation of osteoclasts, the number of Treg cells in synovial tissue increased, the number of Treg cells in peripheral blood decreased, and dysfunction. Van Amelsfort et al. found that Treg cells in peripheral blood and synovial fluid of RA patients increased, and the latter was higher. Treg cells decrease in synovial fluid but Th17 cells increase in RA patients [10]. The number and proportion of Treg cells in peripheral blood of the patients with chronic RA were not statistically different from the control group [11]. Kawashiri et al. found that RA patients not only had reduced Treg cells, but also had defects in immunosuppressive function. Treg cells decreased in patients with active RA, but there was no statistically significant difference in remission compared with the control group, so changes in the number of Treg cells in peripheral blood can monitor changes in RA. The Th17/Treg ratio of healthy people decreased, while RA patients increased significantly, especially during the active phase [12]. The higher the ratio, the more severe arthritis. Meta analysis also confirmed that the increase of Th17 cells and the decrease of 
Treg cells in peripheral blood were related to the pathogenesis of RA. The imbalance of Treg/Th17 can lead to patients' immune dysfunction, which was also the main reason for the pathogenesis of RA [13]. Treatment of RA with monoclonal antibody can increase the number of Treg cells in the peripheral blood, and the number change was negatively correlated with the severity of RA. Möttönen et al found that the number of $\mathrm{CD} 4^{+} \mathrm{CD} 25^{+}$iTreg cells in peripheral blood of RA patients was not significantly different from that of healthy controls, but the number of $\mathrm{CD} 4{ }^{+} \mathrm{CD} 25^{+}$Treg cells in synovial fluid increased significantly. Some date also showed that Treg cells in peripheral blood of RA patients were reduced and Th17 cells and serum IL-17content were increased $[14,15]$, Rosenzwajg et al [16] reported that the proportion of $\mathrm{CD} 4{ }^{+} \mathrm{CD} 25$ high Tregs in peripheral blood of active RA patients was significantly reduced. Lu Xiang and others found that the proportion of $\mathrm{CD} 4{ }^{+} \mathrm{CD} 25^{+}$Treg cells in peripheral blood of patients with active RA was significantly reduced. The levels of IL-17 and TGF- $\beta$ were higher than those in inactive and control groups. Serum IL-17 level was negatively correlated with the proportion of $\mathrm{CD} 4^{+} \mathrm{CD} 25^{+}$Treg cells, and positively correlated with serum TGF-TGF level, suggesting that Th17/Treg imbalance was involved in the occurrence and development of RA, and TGF- $\beta$ involved in the inflammatory response of RA. Astry et al [17] reported that Foxp3 in peripheral blood of RA patients decreased, while IL-17 increased significantly, leading to inflammation and bone destruction. There were also differences in the clinical index research results of Treg cells and RA. Lin Hui et al [18] found that $\mathrm{CD} 4^{+} \mathrm{CD} 25^{+} \mathrm{Foxp}^{+} \mathrm{T}$ cells were not associated with rheumatoid factor titers, erythrocyte sedimentation rate, C-reactive protein (CRP), and disease activity scores of 28 joints. Kawashiri and others believed that $\mathrm{CD}^{+} \mathrm{CD} 25$ highCD127low/Treg cells were negatively correlated with the above indicators. The difference in results may be related to the detection method, the amount of samples included, and the severity of the condition. It was speculated that $\mathrm{CD} 4{ }^{+} \mathrm{CD} 25^{+}$Treg cells played an important role in the occurrence and progression of RA. Due to the decrease in the number of Treg cells or functional defects, which leaded to immune dysfunction, the various cytokines released participated in the immune response, weakened the immune suppression of Treg cells, promoted the increase of inflammatory Th17 cells, and made Th17/Treg cells imbalance, resulting in the occurrence and development of RA and the corresponding changes in the condition. Li Zhiwei et al [19] found that in patients with RA, CD4 was significantly increased, CD8 was significantly decreased, and the $\mathrm{CD} 4 / \mathrm{CD} 8$ ratio was significantly increased. The absolute values of T, B, and NK cells were significantly lower than those of the healthy control group, but B cells and NK cells had no significant changes. At the same time, Treg cells decreased, while Th17 cells increased significantly. Therefore, changes in lymphocyte subsets and Treg/Th17 imbalance were involved in the pathogenesis of RA. Xiang Haiyan et al [20] reported that PBMCs Th17 cells, Th17/Treg ratio and serum IL-17 content of RA patients were significantly increased, Treg cells ( $\mathrm{CD} 4^{+} \mathrm{FoxP}^{+}{ }^{+} \mathrm{T}$ cells) decreased, and microRNA-498 (miR-498) was underexpressed, while STAT3 was highly expressed. After transfection of miR-498 mimic/inhibitor into wild-type STAT3 gene plasmid cells, the expression of STAT3 gene and protein were significantly changed, but the above indicators did not change significantly after transfection of mutant STAT3 gene plasmid cells. In addition, after miR-498 mimic transfection of CD4+T cells, Th17 cells were significantly reduced, and the content of IL-17 was reduced. When co-transfected with pcDNA-STAT3 to $\mathrm{CD}^{+} \mathrm{T}$ cells, the levels of Th17 cells and IL-17 were significantly increased. It indicated that $\mathrm{CD}^{+} \mathrm{T}$ cells in RA patients overexpress miR-498, which can target down-regulation of STAT3 mRNA and protein expression, inhibit Th17 cell differentiation and IL-17 secretion. Lienart etc [21] found that Tregs expressed glycoprotein A repetitive sequence specificity (glycoprotein A repetitions predominant, GARP), transmembrane protein was highly expressed in activated $\mathrm{CD}^{+}$Treg cells, while except activated Th cells. GARP was a potential TGF-TGF binding protein, closely related to the activation of TGF-TGF, which can promote the activation and secretion of TGF-TGF, CD25 ${ }^{+} \mathrm{GARP}^{+}$Treg cells were more immunosuppressive than $\mathrm{CD} 25^{+} \mathrm{GARP}-T$ reg cells. Bai Lijie et al [22] observed the effect of GARP activated Tregs cells on RA and found that RA patients with IFN- $\gamma$, IL-17A content, Th1/Th2 index, anti-CCP, RF, CRP content and ESR were significantly increased, but GARP ${ }^{+}$Tregs, TGF- $\beta 1$, FoxP3 mRNA and IL-4 content were significantly reduced, and CRP content and ERS were significantly negatively correlated with the number of GARP+Tregs in peripheral blood, and were significantly positively correlated with Th1/Th2 index. It suggested that the ratio of GARP+Tregs may be used as a new immunological indicator for the diagnosis of RA. At the same time, TGF-TGF 1 and IFN-TGF significantly increased and IL-4 decreased, indicating that $\mathrm{GARP}^{+}$Tregs had the effect of regulating Th1/Th2 cell to $\mathrm{Th} 2$ in the inflammatory response with $\mathrm{CD}^{+} \mathrm{CD} 25^{+}$Foxp $^{+}{ }^{+}$Tregs. GARP ${ }^{+}$Tregs may be involved in the occurrence and development of RA by regulating the balance of Th1 / Th2 cells to Th2, itself or Th17 in the pathogenesis.

\subsection{Treg Cells and Systemic Lupus Erythematosus (SLE)}

SLE is a common non-organ specific autoimmune connective tissue disease, which is characterized by the production of multiple autoantibodies against double-stranded DNA, and the formation of immune complexes, resulting in multiple organ damage. Most patients have typical butterfly or discoid erythema on their cheeks. Lupus nephritis (LN) is one of the most common and serious complications of SLE and has become the main cause of end-stage renal disease. The incidence of SLE is related to genetic, environmental, estrogen, infection and other factors, and its pathogenesis is not yet clear. After the Treg cells of SLE patients were cultured with autologous Teff, their inhibitory function weakened, but after being cultured with normal human Teff, the inhibitory function of Treg cells was not affected, confirming that Treg cells cannot effectively suppress Teff [23]. The results of research on the number and function of Treg cells in patients with SLE are controversial. Most believed that the number of Treg cells in peripheral blood of SLE patients was significantly reduced, and some believed that the number of Treg cells was reduced or the function was impaired. Also Treg and Teff in a number of SLE patients have no abnormal change in vitro inhibitory. 
Treg has nothing to do with SLE pathogenesis. There is no statistically significant difference in the number and function of normal people. The different results of the study may be related to the included SLE in different stages, ethnic differences and drug effects. Lee et al. reported that $\mathrm{CD} 4{ }^{+} \mathrm{CD} 25^{+}$Treg cells in peripheral blood of SLE patients decreased by $22.1 \%$ compared with the normal control, and their inhibitory function decreased. A recent study [24] found that CD25-T cells and CD25-FoxP3 + cells increased in patients with SLE. The experiments in vitro confirmed that these cells were different from conventional Treg cells and could not effectively suppress Teff. Jia Huiyu and Cheng Guanghui [24] found that Treg cells and TGF- $\beta$ in patients with active SLE were lower than those in inactive and control groups, and the ratio of Th17 cells, Th17 / Treg and IL-6, IL-17, IL-23 were higher in inactive phase and control group. Treg cells in inactive phase of SLE patients were lower than control group. Th17 cells, Th17/ Treg ratio and IL-6, IL-17, IL-23 were higher than control group, indicating that TLE cells were in peripheral blood of SLE patients. The decrease and increase of Th17 cells lead to the imbalance of Treg/Th17 and abnormalities of related cytokines, which were involved in the pathogenesis of SLE, and their growth and decline were related to the activity of SLE. Treatment of SLE with curcumin can significantly reduce Th17 and upregulate the proportion of Treg cells. Huang Keke and others [25] applied AG490 immunosuppressants to block the STAT3 signaling pathway, which could affect the Th17/Treg balance. As a result, Th17 cells in SLE patients increased significantly, while Treg cells decreased significantly, and as the concentration of AG490 increased, the Th17/Treg ratio, IL-17, IL-6 content gradually decreased, while IL-10, TGF- $\beta$ levels increased significantly. After treatment with different concentrations of AG490, STAT3, ROR- $\gamma$ t mRNA levels and STAT3 protein content were significantly reduced, while Foxp3 mRNA levels were significantly increased. It suggested that blocking the STAT3 signaling pathway shifted the Th17/Treg balance to Treg, induced Treg differentiation, inhibited Th17 differentiation, and regulated Th17/Treg balance and ROR- $\gamma \mathrm{t}$, Foxp3 levels. Hu Wei et al [26] detected the expression levels of Th1/Th2/Th17/Treg in peripheral blood of patients with SLE, and found that Th17 and Treg cells in SLE patients were significantly increased, which was significantly positively correlated with CRP levels in patients, and the number of Th17 cells was associated with SLE disease. Activity scores were significantly negatively correlated, but there was no significant difference between Th1 and Th2 expression levels and Th1/Th2 and Th17/Treg in SLE patients and healthy controls. It suggested that Th17/Treg imbalance was involved in the occurrence and development of SLE. Th17 can reflect the activity of SLE disease and provide theoretical and experimental basis for finding new SLE therapeutic targets and therapeutic drugs. Hao Huiqin et al [27] reported that Th17 cells and IL-17 levels in SLE patients were significantly increased, and it was significantly higher in the active phase than in the inactive phase, while Treg cells were significantly reduced, it was significantly lower in the active phase than in the inactive phase, In SLE active phase Th17/Treg was significantly higher than in inactive period and normal control group. However, there was no statistically significant difference in TGF- $\beta$ level between SLE active period, inactive period and normal control group, indicating that Th17/Treg cells participated in the occurrence and development of SLE. And Th17/Treg can be used as a reliable indicator to judge the activity and condition evaluation of SLE disease. The above-mentioned research results indicate that Treg/Th17 imbalance plays an important role in the pathogenesis of SLE. Upregulation of Treg and down-regulation of Th17 cells through drugs or immunosuppressants provide new ideas for the research and treatment of the pathogenesis and immunological mechanism of SLE.

\subsection{Treg Cells and Inflammatory Bowel Disease (IBD)}

IBD is a non-specific colon inflammation, including Crohn 's disease (CD) and ulceralive colitis (ulceralive colitis, UC). Abnormal immune function of intestinal mucosa may be involved in the pathogenesis of IBD, but the etiology and pathogenesis are not yet clear. Research suggested that genetic, environmental and immune factors were involved in the development of $\mathrm{CD}$, and immune dysfunction was one of the key factors. Normally, Th1/Th2 balance disorder was involved in the pathogenesis of IBD [28]. Treg cells mainly played a negative feedback role in suppressing intestinal mucosal immunity. Th1, Th2 and Th17 in the intestinal mucosa can activate Treg cells and produce high levels of IL-10 and TGF- $\beta$ which maintain Th1/Th2 balance during the pathogenesis of IBD by inhibiting Th1 and Th2 cells [29]. Treg cells of intestinal mucosa can also perform two-way regulation and transform with Th17 cells, and form an even more important Th17/Treg cell immune balance with Th17 cells during IBD. In recent years, Th17/Treg cell imbalance theory has become a research hotspot. Treg is a subpopulation of cells with the function of inhibiting Teff proliferation and activation, and can reduce intestinal inflammation in patients with IBD. Chao et al [30] believed that Th17 cells can mediate the occurrence of chronic inflammation and AID, and transform with Treg cells. Therefore, Th17/Treg balance is an important factor for maintaining intestinal immune homeostasis and plays an important role in the development of $\mathrm{CD}$ intestinal inflammation [31], and its imbalance may be one of the causes of IBD. In the UC model, it was found that the number of Thl7 cells in the peripheral blood of mice increased, while the number of Treg cells decreased [32]. It can significantly improve the clinical symptoms of diarrhea mice and IBD by increasing the secretion of anti-inflammatory factors such as IL-10 and TGF- $\beta$, inhibiting the cascade of intestinal inflammation and amplifying the response [33]. Th17 cells infiltrate in the intestinal mucosa of IBD patients. IL-17A, IL-6, IL-1 $\beta$ and Foxp3 are highly expressed in the intestinal mucosa of IBD patients, and the content of IL-17 is increased, while TGF- $\beta$ is only highly expressed in UC. Foxp3+Treg cells which highly expressed $\mathrm{CD} 4^{+} \mathrm{CD} 25^{+}$in peripheral blood decreased 
and Th17 cells increased, resulting in a significant increase in Th17/Treg ratio. IL- $17^{+} \mathrm{IFN} \gamma^{+} \mathrm{T}$ cells and IL- $17^{+} \mathrm{Foxp} 3^{+} \mathrm{T}$ cells are expressed in the colonic mucosa of UC patients. Treg cells in the body are easily polarized into IL-17 secreting cells, and IL- $17^{+}$Foxp $3^{+} \mathrm{T}$ cells can inhibit $\mathrm{T}$ cells activation and stimulation of colonic mucosal inflammation [34]. It suggested that the imbalance of Th17/Treg ratio is related to the development of IBD. Peripheral $\mathrm{CD} 4^{+} \mathrm{CD} 25$ hiFoxp3+Treg cells in active IBD patients decreased, but increased in the remission period, suggesting that Treg cells have an immuno-inflammatory protective role in the formation of intestinal inflammation in IBD. Zhao Xiaojing et al [35] found that Treg cells in peripheral blood of patients with mild to moderate active $\mathrm{CD}$ were significantly lower than those in remission and control group. The decrease of Treg cells was related to the activity of CD, while Th17 cells were higher than those in remission and Th17/Treg Changes were negatively correlated with inflammatory indicators reflecting $\mathrm{CD}$ activity such as erythrocyte sedimentation rate, CRP level and CDAI score, and serum CRP levels were positively correlated with CD disease activity, which could reflect $\mathrm{CD}$ inflammatory activity [36], suggesting Th17/Treg cell imbalance Participate in the development of CD intestinal inflammation. The above research shows that Th1/Th2 and Th17/Treg and their cytokines are involved in the occurrence and development of IBD, and are closely related to the disease.

\subsection{Treg Cells and Autoimmune Thyroid Diseases (AITD)}

AITD is a common organ-specific AID in human and the most common thyroid disease than other iodine deficiency disease, and its incidence has increased in recent years. The study found that $90 \%$ of thyroid diseases are AITD, mainly including diffuse toxic goiter (Graves' Disease (GD disease) and Hashimoto thyroiditis (HT), characterized by hyperthyroidism and hypothyroidism, Its common feature is the deficiency of immune tolerance to its own thyroid tissue, which is often accompanied by a variety of autoantibody positive and lymphocyte infiltration. The causes of AITD are complex, but have similar genetic and immunological foundations. Prolonged exposure to environmental triggers can promote disease in people with specific genetic backgrounds. In the past, studies on the correlation between AITD and Th1/Th2 subgroup and cytokines have been extensively carried out, and it is believed that the pathogenesis of AITD is closely related to Th1 and Th2. With the deepening of the research, it is found that the traditional Thl/Th2 cell imbalance still cannot well explain the pathogenesis of AITD. Wang et al. [37] used female rhesus monkeys to simulate the human GD model for the first time and found that Treg cells decreased and Th17/Treg ratio increased. Thymusectomy in newborn mice eliminates peripheral $\mathrm{CD} 4^{+} \mathrm{CD} 25^{+}$Treg cells, which can trigger a variety of organ-specific AIDs, and may inhibit the autoimmunity caused by serum thyroglobulin with $\mathrm{CD} 4{ }^{+} \mathrm{CD} 25^{+}$Treg cells Response. Marazuel et al. reported that the number of peripheral blood Treg cells in AITD patients was significantly higher than that in the control group, while the inhibitory ability was significantly reduced. In peripheral blood of AITD patients, T cells expressing Foxp3 and GITR increase, but their ability to inhibit cell proliferation decreases, and most of them cannot down-regulate the autoimmune response and reduce the damage of inflammation to thyroid tissue [38]. The number of $\mathrm{CD} 4^{+} \mathrm{CD} 25^{+}$Treg decreased in AITD patients, especially Foxp3 $+\mathrm{CD} 4^{+} \mathrm{CD} 25^{+}$Treg, while the number of activated $\mathrm{CD} 4^{+} \mathrm{T}$ cells increased. Chen Wanzhi found that the level of IL-17AmRNA and serum IL-17 in thyroid patients was significantly higher than those in the control group, and the HT hypothyroidism group was higher than the normal group, while the Foxp3mRNA expression and IL-10 level was significantly lower than the control group. There was no significant difference between the hypothyroidism group and the normal hyperthyroidism group of HT. Mao et al. reported that $\mathrm{CD}^{+} \mathrm{CD} 25^{+} \mathrm{Foxp}^{+}$Treg in peripheral blood of GD patients was significantly reduced, and it was negatively correlated with TSHR antibody titer. The immunosuppressive ability of Treg cells in GD patients was significantly reduced, but the number was not significantly different from that in the control group, indicating that the abnormal function of Treg cells is closely related to the pathogenesis of GD. Abnormal number and/or function of Treg can make the patient's Treg mismatch with the activity of self-reactive $\mathrm{T}$ cells and cause disease. Kahaly et al. found that the immunosuppressive capacity of Treg cells in peripheral blood of GD patients was significantly lower than that of the control group, but the number was not significantly different from that of the control group, suggesting that the decreased function of Treg cells is closely related to the incidence of GD. Tang Dahai et al also found the same results by the detection of $\mathrm{CD}^{+}$and $\mathrm{CD} 25^{+}$Treg cells in peripheral blood of GD patients. Glick et al [39] used FCM to measure the number of Tregs in peripheral blood of patients with AITD, and found that Treg CD4 ${ }^{+} \mathrm{T}$ cells had no significant difference from the control group, but the inhibitory ability decreased, suggesting that Treg cells in AITD patients have reduced function. After treatment with methimazole in adolescent GD patients, the percentage and absolute value of Treg in peripheral blood increased [40]. Li Zhongyuan etc [41] reported the number of $\mathrm{CD} 4{ }^{+} \mathrm{CD} 25^{+}$Treg and the level of Foxp3 mRNA in peripheral blood of elderly patients with GD were significantly lower, the $\mathrm{CD} 4{ }^{+} \mathrm{CD} 25^{+}$Treg percentage and Foxp 3 mRNA expression level with the illness alleviates obviously rise after the treatment of the iodine 131 in 3 months, and is better than a sulfhydryl imidazole group. However, there was no statistically significant difference in the Foxp3 mRNA expression level between the methimazole treatment group and before treatment. Prompt iodine 131 may increase the number of $\mathrm{CD} 4{ }^{+} \mathrm{CD} 25^{+}$cells. The number of Treg cells and the level of Foxp3 mRNA can improve the immune tolerance of elderly GD patients and affect the metabolic level of thyroid hormones. The abnormality of Treg quantity and function is related to the pathogenesis and prognosis of GD. 
The immunosuppressive ability of Treg cells in thyroid tissue and peripheral blood of HT patients is reduced, while the Th17 cell response is enhanced. Treg/Th17 immune imbalance may be involved in the occurrence and development of HT. The above-mentioned research shows that the reduction of Treg cells in AITD patients is more common and accompanied by dysfunction, but there are also reports of increased Treg numbers and no obvious changes, which may be related to the detection method, the number of cases, and the degree of disease.

\subsection{Treg Cells and Multiple Sclerosis (MS)}

MS is a T cell-mediated, chronic progressive demyelinating inflammatory autoimmune disease of the central nervous system caused by immune tolerance disorders. The patients are mostly young adults, and the clinical manifestations are fatigue, ataxia and cognitive impairments. If you do not intervene in time, it will affect their ability to take care of themselves, causing a serious financial burden on the family [42]. The pathogenesis of MS is still unclear, and factors such as infection, immunity, environment, and genetics play an important role in the pathogenesis of MS. It is traditionally believed that the onset of MS is mainly related to the dysfunction of Th1 and Th2 cells, and the abnormal increase of Th1 cells is the main immune mechanism of MS. Manni [43] found that experimental autoimmune encephalomyelitis (EAE) Th1 cells in mice increased, and the level of autocrine IL-12 increased, suggesting that Th1 cells are involved in its pathogenesis. But clearing IL-12 or blocking the binding of IL-12 to its receptors, mice are still susceptible to EAE [44]. EAE is a good animal model for studying MS, and its pathogenesis is highly similar to MS, confirming that Th17 cells are an important pathogenic factor for MS, and Treg function blockade is positively correlated with the degree of disease deterioration. The brain and spinal cord tissues of EAE rats are infiltrated by a large number of inflammatory cells, and inflammatory cell sheaths are formed around the blood vessels, showing typical EAE pathological characteristics. Klei-Newietfeld et al [45] reported that the number of Tregs and /or dysfunction in patients with MS, and the abnormal Foxp3 signaling and protein expression of Treg in patients with relapsing-remitting multiple sclerosis, lead to decreased Treg function and promote the occurrence of disease. Li Dan [46] used flow cytometry and flow microsphere chip capture technology to detect the content of $\mathrm{T}$ lymphocyte subsets and plasma cytokines, and found Th1, Th17 cells and Th17/Treg ratio and IFN- $\gamma$, IL-17A in MS patients were significantly increased, while the contents of Treg, Tc1 cells and IL-4 and IL-10 were significantly reduced. Compared with the MS group, the contents of Tc1, Tc2, Treg cells and IL-4 and IL-10 in the prednisone treatment group were significantly increased, while Th1, Th17 cells, Th17/Treg ratio and IL-17A content were significantly reduced. Blocking the signaling pathway of heparin binding cytokines in patients, the number of Tregs decreased and /or the abnormal function and EAE symptoms of MS patients were significantly improved without any adverse reactions. Jiang Juean et al [47] found that the number of $\mathrm{CD}^{+} \mathrm{CD} 28^{-} \mathrm{T}$ cells increased in MS patients, while $\mathrm{CD}^{+} \mathrm{CD} 28^{-} \mathrm{T}$ cells decreased, and $\mathrm{CD} 45 \mathrm{RO}$ are overexpressed in $\mathrm{CD} 4^{+} \mathrm{CD} 28^{-} \mathrm{T}$ cells, while the expression of CD45RO, CD25 and CD45RA in CD4 ${ }^{+} \mathrm{CD} 28^{-} \mathrm{T}$ cells were all reduced, and the $\mathrm{CD} 4^{+} \mathrm{CD} 28^{-} \mathrm{T}$ cells of MS patients in relapse stage were significantly increased, while the $\mathrm{CD} 8^{+} \mathrm{CD} 28^{-} \mathrm{T}$ cells were significantly decreased. The above research shows that the occurrence of MS is closely related to the imbalance of Th17/Treg and Th1/Th2 cytokines. Cytokines such as IL-6 and IL-23 are involved in the pathogenesis of MS and are closely related to the severity of MS. Th17 upregulation or Treg downregulation participates in the occurrence and development of MS. Targeting downregulation of Th17 or upregulation of Treg for MS can correct the imbalance of Th17/Treg ratio. It has potential therapeutic value and provides new immunological treatment options for MS from the perspective of Treg.

\subsection{Treg Cells and Primary Sjogren's Syndrome (PSS)}

PSS is a chronic inflammatory autoimmune disease that mainly invades exocrine glands such as salivary and lacrimal glands. It is one of the most common diffuse connective tissue diseases in China. It is characterized by high lymphocyte infiltration and common symptoms are obvious Dry eyes, accompanied by other systemic damage, seriously affect the patient's quality of life. The pathogenesis of SS is complex, which may be due to genetic, environmental, infection, and abnormal sex hormones and other factors, resulting in immune systemic dysfunction, inflammatory mediators infiltration caused tissue damage, and then developed into SS. Sudzius et al. believed that the imbalance of peripheral blood $\mathrm{T}$ cell differentiation was an important factor that caused the onset of SS. Zhu Jiandong et al [48] found that the lip gland tissue and serum IL-17 levels in SS patients were significantly higher than those in the control group, and Foxp3m RNA expression in the lip gland tissue was reduced. Alunno et al. [49] reported that the peripheral blood Treg cells of pSS patients were slightly lower than the control group, and the labial gland tissue contained a large amount of $\mathrm{CD} 4{ }^{+} \mathrm{CD} 25^{+}$low $\mathrm{T}$ cells, which was similar to the results of other AID target organs with Treg cells higher than peripheral blood. It is speculated that it may be to suppress self-reactive $\mathrm{T}$ cells, regulate the local immune response, and accumulate Treg in the inflammation area of the target organ as a reverse regulation or negative feedback mechanism. Wang Qikai [50] found that the spleen $\mathrm{CD}^{+}{ }^{+} \mathrm{Foxp}^{+} \mathrm{T}$ cells/CD4 ${ }^{+} \mathrm{T}$ cells of SS NOD model rats were significantly increased, and the costimulatory molecule B7-H4-Fc group was more $\mathrm{CD} 4^{+} \mathrm{IFN}$ than $\mathrm{IgG} 2 \mathrm{a}-\mathrm{Fc}$ group, baseline group and NS group $-\gamma^{+} \mathrm{T}$ cells $/ \mathrm{CD}^{+} \mathrm{T}$ cells were significantly reduced, but there was no significant difference between the last three groups of $\mathrm{CD} 4^{+} \mathrm{IL}-17^{+} \mathrm{T}$ cells $/ \mathrm{CD} 4^{+} \mathrm{T}$ cells, suggesting that $\mathrm{B} 7-\mathrm{H} 4$ protein can delay the infiltration of submandibular gland lymphocytes in NOD mice, Reduce the level of inflammatory cytokines and inhibit the progress of 
autoimmune inflammation. This is basically the same as Podojil and other applications of B7-H4Ig in the treatment of EAE mice, which significantly increased the number of central nervous system Treg cells and significantly enhanced the function. It is confirmed that there is $\mathrm{B} 7-\mathrm{H} 4$ protein expression in the submandibular gland of NOD mice. When $\mathrm{B} 7-\mathrm{H} 4$ protein is administered, it can delay the pathological progress of the submandibular gland of NOD mice, inhibit the differentiation of Th1 and Th17, upregulate Treg cells, and provide clinical treatment for SS with B7-H4 protein. Theoretical basis. Notch signaling pathway mainly regulates the differentiation of Treg cells, mediates immunosuppression, and reduces the number of Treg cells to mediate immune responses, exacerbating immune inflammation. Inhibiting Notch signaling can promote Treg to secrete the anti-inflammatory cytokine IL-10 and inhibit the expression of pro-inflammatory cytokine IL-1 $\beta$ [51]. Wang Kun et al [52] found that IL-2 and IL-17 increased in pSS model rats, and IL-5 and peripheral blood $\mathrm{CD} 4{ }^{+} \mathrm{CD} 25^{+}$Treg, $\quad \mathrm{CD} 4^{+} \mathrm{CD} 25^{+} \mathrm{Notch}^{+}$cells and $\mathrm{CD} 4^{+} \mathrm{CD} 25^{+}$Notch $^{+} \mathrm{T}$ cells expressed The expression of Notch3, Delta1, Jagged2 mRNA and protein in lung tissue was significantly increased, suggesting that $\mathrm{CD} 4^{+} \mathrm{CD} 25^{+}$Treg cells have an inhibitory effect on the inflammatory response in pSS rats. Notch pathway promotes the reduction of pSS lung function by regulating Treg expression.

\subsection{Treg Cells and Psoriasis}

Psoriasis, commonly known as psoriasis (Psoriasis, Ps), is a chronic immune skin disease characterized by keratinocyte hyperplasia, inflammatory cell and $\mathrm{T}$ lymphocyte infiltration. Patients often have red, scaly, or white patches on their skin, dry, Itching or cracking and other symptoms, about $1 / 3 \mathrm{Ps}$ patients can develop psoriatic arthritis (Psoriatic arthritis, PsA). Peripheral blood Treg cells increase in Ps patients and are related to the degree of disease activity. From asymptomatic to skin lesions, $\mathrm{CD}^{+}, \mathrm{CD}^{+}, \mathrm{Foxp}^{+}$cells gradually increase, and the $\mathrm{Treg} / \mathrm{CD}^{+}{ }^{+} \mathrm{T}$ ratio at the lesion site is much higher than that at the non-lesion site [53]. The occurrence of Ps and the degree of lesions is related to the decrease of Foxp3 mRNA levels. Foxp $3^{+}$Treg cells are reduced in the skin lesions of advanced patients. In severe cases, there are even IL- $17 \mathrm{~A}^{+} / \mathrm{Foxp}^{+} / \mathrm{CD}^{+}$triple positive cells in local lesions, indicating the degree of lesions. It is negatively correlated with the number of Foxp $3^{+}$Treg cells. Children with seizures had significantly increased Th17 and Foxp3+Treg cells and Th17/Treg cell ratios, and were positively correlated with the degree of inflammation [54]. Treatment of Ps with vitamin D3 can induce DCs in the epidermis and dermis to differentiate into different types of Treg cells, maintain immune tolerance, and thus relieve clinical symptoms. After treatment with infliximab, Treg cells were significantly increased, and clinical symptoms were significantly improved [55]. Therefore, the imbalance of Th17/Treg ratio plays an important role in the pathogenesis of Ps.

\section{Application and Prospect of Treg Cells}

Treg cells are an important subset of $\mathrm{CD} 4^{+} \mathrm{T}$ cells recently discovered, which have a negative immunoregulatory role and participate in maintaining the immune balance of the body. Its discovery breaks the conventional understanding of the immune tolerance mechanism and is increasingly receiving clinical attention. At present, research on Treg cells has been widely carried out at home and abroad, and certain progress has been made in its source and classification, differentiation and regulation, and research in AID. Combined with the research results of Th17 cells, the Th17/Treg immunology theory was formed, which enriched the connotation of the traditional Th1/Th2 immunology theory, expanded the understanding of immune-related diseases, and gradually formed a comprehensive model of Th17/Treg and Th1/Th2 and also opened up a new field of disease immunology research. With the continuous deepening of research on Treg, it has found that its number and function abnormalities participate in the pathogenesis of AID such as RA, SLE, MS, AITD, and its changes are related to the severity of the disease. Using strategies to regulate Treg, targeting AID to upregulate Treg or down- regulate Th17, from the perspective of Treg to provide a new immunological treatment plan for AID, intervention and correction of Treg/ Th17 imbalance has achieved gratifying efficacy. However, because the onset of AID involves Treg/Th17 and Th1/Th2 and their corresponding cytokines and transcription factors, it constitutes a huge and complex immune network system. The interaction and influence of many factors in this system are quite complex issues. The target of many mechanisms is not yet clear, and the research results of Tregs in AID are not very consistent, and controversy has also followed. Therefore, large-scale prospective Tregs research and random double-blind multi-center verification of AID will become the focus of future research. Exploring their interrelationships in disease, the role of target cells and the precise mechanism of immune regulation will help to understand the body's immune status, recognize the precise mechanism of the onset of immune-related diseases, and apply Treg for diagnosis, treatment and prevention AID provides new ideas.

\section{Acknowledgements}

Cangzhou Key R \& D Plan and Guidance Project (Project No: 1833020011).

Natural Science Foundation of Cangzhou Medical College (No. 18Z015).

\section{References}

[1] Pan HF, Leng RX, Wu GC, et al. Advance in epidemiologic studies on major autoimmune diseases. Chin J Dis Control Prev 2018, vol. 22, pp. 1093-1095, 1105.

[2] Liu HP, Cao AT, Feng T, et al. TGF- $\beta$ converts Th1 cells into Th17 cells through stimulation of Runx1 expression [J]. Eur J Immunol. 2015, vol. 45, pp. 1010-1018. 
[3] Zhivaki D, Lemoine S, Lim A, et al. Respiratory Syncytial Virus Infects Regulatory B Cells in Human Neonates via Chemokine Receptor CX3CR1 and Promotes Lung Disease Severity [J]. Immunity. 2017, vol. 46, pp. 301-314.

[4] Sawant DV, Hamilton K, Vignali DA. Interleukin-35: Expanding Its Job Profile [J]. J Interferon Cytokine Res. 2015, vol. 35, pp. 499-512.

[5] Castellani ML, Anogeianaki A, Felaco P, IL-35, an anti-inflammatory cytokine which expands CD4+CD25+ Treg Cells [J]. J Biol Regul Homeost Agents. 2010, vol. 24, pp. 131-135.

[6] Bantug G, Galluzzi L, Kroemer G, et al. The spectrum of T cell metabolism in health and disease [J]. Nat Rev Immunol, 2018, vol. 18, pp. 19-34.

[7] Noack M, Miossec P. Th17 and regulatory T cell balance in autoimmune and inflammatory diseases $[\mathrm{J}]$. Autoimmun Rev, 2014, vol. 13, pp. 668-677.

[8] Zhang L, Wang YX, Yang T, et al. A current review ofthe relationship between IL-12 and autoimmune diseases. Chin J Dis Control Prev, 2014, vol. 18, pp. 1099-1103

[9] Nie H, Zheng Y, Li R, et al. Phosphorylation of FOXP3 controls regulatory $\mathrm{T}$ cell function and is inhibited by TNF- $\alpha$ in rheumatoid arthritis [J]. Nat Med, 2013, vol. 19, pp. 322-328.

[10] Moradi B, Schnatzer P, Hagmann S, et al. CD4+CD25+/highCD127low/- regulatory $\mathrm{T}$ cells are enriched in rheuma-toid arthritis and osteoarthritis joints--analysis of frequency and phenotype in synovial membrane, synovial fluid and peripheral blood [J]. Arthritis Res Ther. 2014, vol. 16, pp. R97.

[11] Walter GJ, Fleskens V, Frederiksen KS, et al. Phenotypic, Functional, and Gene Expression Profiling of Peripheral CD45RA+and CD45RO+CD4+CD25+CD127 (low) Treg Cells in Patients With Chronic Rheumatoid Arthritis [J]. Arthritis Rheumatol, 2016, vol. 68, pp. 103-116.

[12] Wang W, Shao S, Jiao Z, et al. The Th17/Treg imbalance and cytokine environment in peripheral blood of patients with rheumatoid arthritis [J]. Rheumatol Int, 2012, vol. 32, pp. 887-893.

[13] Liu YX, Zhu YF, Liu Y, et al. Quantity of Th17 and Treg cells in the peripheral blood from patients with rheumatoid arthritis: a meta analysis. J Zhengzhou Univ (Med Scie). 2015, vol. 50, pp. 606-610.

[14] Dong L, Wang X, Tan J, et al. Decreased expression of microRNA-21 correlates with the imbalance of Th17 and Treg cells in patients with rheumatoid arthritis [J]. J Cell Mol Med. 2014, vol. 18, pp. 2213-2224.

[15] Jain M, Attur M, Furer V, et al. Increased plasma IL-17F levels in rheumatoid arthritis patients are responsiveto methotrexate, anti-TNF, and T cell costimulatory modulation [J]. Inflammation. 2015, vol. 38, pp. 180-186.

[16] Rosenzwajg M, Lorenzon R, Cacoub P, et al. Immunological and clinical effects of low-dose interleukin-2 across 11 autoimmune diseases in a single, open clinical trial [J]. Ann Rheum Dis. 2019, vol. 78, pp. 209-217.

[17] Astry B, Venkatesha SH, Moudgil KD. Involvement of the IL-23/IL-17 axis and the Th17/Treg balance in the patho-genesis and control of autoimmune arthritis [J]. Cytokine. 2015, vol. 74, pp. 54-61.

[18] Lin H, Zhang GD, Tang HH, et al. The Change of CD4+CD25+ Regulatory T Cells in Patients with Rheumatoid Arthritis. J Sichuan Univ (Med Sci Edi). 2014, vol. 45, pp. 618-622.

[19] Li ZW, Tian YF Lu PP, et al. Detection and significance of lymphocyte and tregth 17 balance in patients with Rheum-atoid Arthritis. Int J Lab Med. 2019, vol. 74, pp. 2026-2028.

[20] Xiang HY, Pan F, Yan JZ, et al. Upregulation of miR-498 suppresses Th17 cell differentiation by targeting STAT3 in rheumatoid arthritis patients. Acta Physiologica Sinica, 2018, vol. 70 , pp. 167-174.

[21] Liénart S, Merceron R, Vanderaa C, et al. Structural basis of latent TGF- $\beta 1$ presentation and activation by GARP on human regulatory T cells $[\mathrm{J}]$. Science. 2018, vol. 362, pp. 952-956.

[22] Bai L, Wu Y, Li HB, et al. The effects of GARP activated Tregs cells on rheumatoid arthritis and its mechanism J Practical Med, 2019, vol. 35, pp. 2276-2280.

[23] Von Spee-Mayer C, Siegert E, Abdirama D, et al. Low-dose interleukin-2 selectively corrects regulatory $\mathrm{T}$ cell defects in patients with systemic lupus erythematosus [J]. Ann Rheum Dis. 2016, vol. 75, pp. 1407-1415.

[24] Jia HY, Cheng GH. Expressions and Significance of Th17 Treg Cell and Related Cytokines in Peripheral Blood in Patients with SLE. Hebei Med, 2018, vol. 24, pp. 1277-1280.

[25] Huang KK, Zhou CY, Yan ZT, et al. Application of AG490 to Regulation Th17/Treg Balance in Systemic Lupus Erythematosus by Blocking STAT3. Med Innovation China, 2019, vol. 16, pp. 141-145

[26] $\mathrm{Hu} \mathrm{W}$, Wang S, Wang X, et al. Expression of Th1 /Th2 /Th17 /Treg in peripheral blood of patients with SLE and its clinical significance. Southeast Univ ( Med Sci Edi). 2019, vol. 38, pp. 308-312.

[27] Hao HQ, Zhang SR, Li XF, et al. Value of determining Th17 and regulatory $\mathrm{T}$ cells in the peripheral blood in evaluating patients with systemic lupus erythematosus. Chin J Prac Inter Med, 2018, vol. 38, pp. 242-244.

[28] Li J, Ueno A, Fort Gasia M, et al. Profiles of la mina propria T helper cell subsets discri minate between ulcerative colitis and crohn' s disease [J]. Inflamm Bowel Dis, 2016, vol. 22, pp. 1779-1792.

[29] Iboshi Y, Nakamura K, Ihara E, et al. Multigene analysis unveils distinctive expression profiles of helper T-cell related genes in the intestinal mucosa that discri minate between ulcerative colitis and crohn's disease [J]. InflammBowel Dis, 2014, vol. 20, pp. 967-977.

[30] Chao K, Zhang S, Yao J, et al. Imbalances of CD4 (+) T-cell subgroups in Crohn's disease and their relationship with disease activity and prognosis [J]. J Gastroenterol Hepatol, 2014, vol. 29, pp. 1808-1814.

[31] D'Ambrosio A, Cossu A, Amendola A, et al. Lamina propria $\mathrm{CD} 4+\mathrm{LAP}+$ regulatory $\mathrm{T}$ cells are increased in active ulcerative colitis but show increased IL-17 expression and reduced suppressor activity [J]. J Crohns Colitis, 2016, vol. 10, pp. 346-353. 
[32] $\mathrm{Xu} \mathrm{Z}$, Wei $\mathrm{C}$, Zhang RU, et al. Epigallocatechin-3-gallate-induced inhibition of interleukin-6 release and adjustment of the regulatory $\mathrm{T} / \mathrm{T}$ helper 17 cell balance in the treatment of colitis in mice [J]. Exp Ther Med. 2015, vol. 10, pp. 2231-2238.

[33] Wang CYL, Sun JG, Geng Y, et al. Progress of Treg/Thl7 immune axis imbalance in ulcerative colitis and its detection by them ethod of flow cytometry. J Immuno. 2015, vol. 31, pp 541-544.

[34] Lee SY, Lee SH, Yang EJ, et al. Metformin ameliorates inflammatory bowel disease by suppression of the STAT3 signaling pathway and regulation of the between Th17/Treg balance. PLoS One, 2015, vol. 10, pp. e135858.

[35] Zhao XJ, Ma JJ, Zhu YJ, et al. Alterations of Th17/Treg cells in peripheral blood and serum inflammatory biomarkers in patients with Crohn's disease and its clinical significance. J Nanjing MedUniv (Nat Scie) 2017, vol. 37, pp. 1000-1004.

[36] Alper A, Zhang L, Pashankar DS. Correlation of erythrocyte sedimentation rate andC-reactive protein with pediatric.

[37] inflammatory bowel disease activity. J Pediatr Gastroenterol Nutr, 2017, vol. 65, pp. e25-27.

[38] Wang $\mathrm{Y}, \mathrm{Wu} \mathrm{LP}, \mathrm{Fu}$ J, et al. Hyperthyroid monkeys:a nonhuman primate model of experimental Graves'disease. J Endocrinol, 2013, vol. 219, pp. 183-193.

[39] Bao QY, Xu YS, Yang HY. Regulatory T Cells and Endocrine Diseases. Medical Recapitulate. 2014, vol. 20, pp. 3278-3280.

[40] Glick AB, Wodzinski A, Fu P, et al. Impairment of regulatory $\mathrm{T}$ cell function in autoimmune thyroid disease. Thyroid, 2013, vol. 23, pp. 871-878.

[41] Klatka M, Grywalska E, Partyka M, et al. Th17 and Treg cells in adolescents with Graves'disease, Impact of treatment with methimazole on these cell subsets. Autoimmunity, 2014, vol. 47, pp. 201-211.

[42] Li ZY, Li CX, Cui LR, et al. Transformation analysis of peripheral blood CD4+ CD25+ Treg, Foxp3 mRNA level and biochemical index of elderly patients with Graves before and after treatment with Iodine-131 and methimazole. Chinese $\mathrm{J}$ Control Endemic Diseases, 2018, vol. 33, pp. 617-621.

[43] Biglar Chopoghlou S, Hanifi N, Varjoshani NJ. A comparative study of uncertainty and coping strategies of patients with multiple sclerosis for members and nonmembers in peer groups. Health Care Women Int. 2019, vol. 1, pp. $1-14$.

[44] Ravyn D, Goodwin B, Lowney R, et al. Continuous learning in multiple sclerosis care: a qualitative study of the expanded learning model for systems. Int J Med Educ. 2019, vol. 10, pp. 122-128.
[45] Lamers I, Raats J, Spaas J, et al. Intensity-dependent clinical effects of an individualized technology-supported task-oriented upper limb training program in Multiple Sclerosis: A pilot randomized controlled trial. Mult Scler Relat Disord. 2019, vol. 34, pp. 119-127.

[46] Kleinewietfeld M, Hafler DA. Regulatory $T$ cells in autoimmune neuroin flammation. Immunol Rev. 2014, vol. 259, pp. 231-244.

[47] Li D. Detection of Tlymphocyte subsets and related cytokines in 31 cases of multiple sclerosis. J Tianjin Medi University. 2019, vol. 25 , pp. 80-83.

[48] Jiang JA, Liu CP, Xue Q, et al. Changes and clinical significance of peripheral CD28-T cell subsets in patients with multiplesclerosis. Chin J Neuroimmunol \& Neurol, 2019, vol. 26, pp. 391-396.

[49] Zhu JD, Wang ZQ, Zhang PN. The Imbalance of Immunity Between Th17 and CD4+CD25+Regulatory T Cell in Patients with Sjgren's Syndrome. J Radio Immuno, 2012, vol. 25, pp. 301-303.

[50] Alunno A, Petrillo MG, Nocentini G, et al. Characterization of a new regulatory $\mathrm{CD}+4 \mathrm{~T}$ cell subset in primary Sjgren' $\mathrm{s}$ syndrome. Rheumatology (Oxford), 2013, vol. 52, pp. 1387-1396.

[51] Wang QK, Yuan X, Zhou YB, et al. Effect of costimulatory molecules B7-H4 on T lymphocyte subsets of NOD mice in Sjgren's syndrome. Chin J Dis Control Prev. 2018, vol. 22, pp. 731-735.

[52] Li H, Wang L, Pang Y, et al. In patients with chronic aplastic anemia, bone marrowderived MSCs regulate the Treg/Th17 balance by influencing the Notch/RBP-J/FOXP3/ROR $\gamma \mathrm{t}$ pathway. Sci Rep. 2017, vol. 7, pp. 42488.

[53] Wang K, Wan L, Liu J. Relationship between changes of lung function and Notch pathway and regula-tory $\mathrm{T}$ cells in pSS rats. Chin J Immu, 2019, vol. 35, pp. 2191-2194.

[54] Raychaudhuri SK, Saxena A, Raychaudhuri SP. Role of IL-17 in the pathogenesis of psoriatic arthritis and axial spondyloarthritis. Clin Rheumatol. 2015, vol. 34, pp. 1019-1023.

[55] Zhang L, Li Y, Yang X, et al. Characterization of Th17 and FoxP3 (+) Treg Cells in Paediatric Psoriasis Patients. Scand J Immunol. 2016, vol. 83, pp. 174-180.

[56] Keijsers RR, Joosten I, Hendriks AG, et al. Balance of Treg versus T-effector cells during systemic treatment with adalimumab and topicaltreatment with calcipotriol-betamethasone dipropionate ointment. Exp Dermatol. 2015, vol. 24, pp. 65-67. 